International Journal of Pure and Applied Mathematics

Volume 104 No. 4 2015, 543-549

ISSN: 1311-8080 (printed version); ISSN: 1314-3395 (on-line version)

url: http://www.ijpam.eu

doi: http://dx.doi.org/10.12732/ijpam.v104i4.6

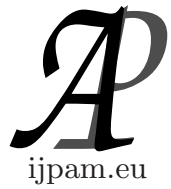

\title{
IDEALS AND THEIR FUZZIFICATIONS IN IMPLICATIVE SEMIGROUPS
}

\author{
Admi Nazra \\ Department of Mathematics \\ Andalas University \\ Kampus Unand Limau Manis Padang, 25163, INDONESIA
}

\begin{abstract}
This research is motivated by the existence of previous research on the ideals of implicative semigroups ([4]), and on the fuzzy set of implicative semigroups $([5],[6])$.

In this paper we study a relation between ideals of implicative semigroups and extended special sets of implicative semigroups. On the other hand the notion of a fuzzy ideal of an implicative semigroup is introduced and its characterization will be studied.
\end{abstract}

AMS Subject Classification: 20M12, 06B10, 03B52, 03E72

Key Words: implicative semigroups, fuzzy sets, fuzzy ideals

\section{Introduction}

The notion of an implicative semigroup is introduced by Chan and Shum [2] as a generalization of an implicative semilattice that was introduced by Nemitz [7]. Then Chan and Shum [2] established some elementary properties of implicative semigroups. Jun and Kim [4] introduced the notion of ideals in implicative

Received: May 28, 2015

(c) 2015 Academic Publications, Ltd. url: www.acadpubl.eu 
semigroups and a special set of an implicative semigroup. Then they provided a condition for the special subset to be an ideal. They also established two characterizations of an ideal. Bang and So [1] introduced, the so called, an extended special set in implicative semigroups and obtained some relations with an ordered filter as a kind of implicative semigroups. On the other hand, Kuresh et al. [6] studied fuzzy sets of implicative semigroups.

In this paper we study two things. First, by using the extended special set, we study its relation with ideals of implicative semigroups. Second, we introduce a fuzzy set, the so called, the fuzzy ideal of implicative semigroups and provide its characterization.

\section{Implicative Semigroups}

In this section we review some definitions and results related to implicative semigroups (see [2] dan [4]). Anegatively partial ordered semigroup (n.p.o semigroup) is a set $S$ with a partial ordering " $\leq$ " and a binary operation "." such that for all $x, y, z \in S$ we have:

(1) $(x \cdot y) \cdot z=x \cdot(y \cdot z)$,

(2) $x \leq y$ implies $x \cdot z \leq y \cdot z$ and $z \cdot x \leq z \cdot y$,

(3) $x \cdot y \leq x$ and $x \cdot y \leq y$.

If there is an additional binary operation $*: S \times S \rightarrow S$ such that for any $x, y, z \in S, z \leq x * y$ if and only if $z \cdot x \leq y$, then an n.p.o semigroup is said to be implicative.

For briefly, an implicative n.p.o semigroup $(S, \leq, \cdot, *)$ is called an implicative semigroup $S$. If an implicative semigrup $S$ satisfies $x \cdot y=y \cdot x$ for all $x, y \in S$ then it is said to be commutative, and if $x *(y * z)=(x * y) *(x * z)$ for all $x, y, z \in S$ then it is said to be left distributive.

Some properties of implicative semigroups $S$ which will be used in this paper are as follows.

Proposition 2.1 ([2]). Let $S$ be an implicative semigroup. Then for any $x, y, z \in S$, the following holds:

(1) $x \leq 1$, with $x * x:=1$, and $x=1 * x$.

(2) $x \leq y$ if and only if $x * y=1$. 
Proposition $2.2([3])$. If $S$ is a commutative implicative semigroup then for every $x, y, z \in S$, we have $x *(y * z)=y *(x * z)$.

Now we recall the definition of ideals of implicative semigroups (see [4]).

Definition 2.3 ([4]). Let $S$ be an implicative semigroup. A subset $I$ of $\mathrm{S}$ is called an ideal of $S$ if

(I1) $x \in S$ and $a \in I$ imply $x * a \in I$,

(I2) $x \in S$ and $a, b \in I$ imply $(a *(b * x)) * x \in I$.

Theorem 2.4 ([4]). Let $S$ be a commutative implicative semigroup. A subset $I$ of $S$ is an ideal of $S$ if and only if it satisfies: (a). $1 \in I$, and (b). $x *(y * z) \in I$ and $y \in I$ imply $x * z \in I$ for all $x, y, z \in S$.

\section{Fuzzy Sets of Implicative Semigroups}

In this section, we review some definitions used in this paper, namely fuzzy ordered filter, fuzzy implicative ordered filter and introduce the notion of fuzzy ideals of implicative semigroups.

Definition 3.1 ([6]). A fuzzy set $\mu$ in an implicative semigroups $S$ is called a fuzzy ordered filter of $S$ if it satisfies the following conditions: C1) $\mu(1) \geq \mu(x)$, for all $x \in S$, and C2) $\mu(y) \geq \min \{\mu(x * y), \mu(x)\}$, for all $x, y \in S$.

Definition $3.2([5])$. A fuzzy set $\mu$ in an implicative semigroups $S$ is called a fuzzy implicative ordered filter of $S$ it satisfies conditions C1) and C3) $\mu(x * z) \geq \min \{\mu(x *(y * z)), \mu(x * y)\}$, for all $x, y, z \in S$.

It was proved by Jun [5], that every fuzzy ordered filter $\mu$ is order preserving, i.e if $x \leq y$ then $\mu(x) \leq \mu(y)$.

Now, we consider the fuzzification of an ideal as in the following definition.

Definition 3.3. Let $S$ be a commutative implicative semigroup. A fuzzy set $\mu$ in $\mathrm{S}$ is called a fuzzy ideal of $S$ if it satisfies conditions C1) and C4) $\mu(x * z) \geq \min \{\mu(x *(y * z)), \mu(y)\}$ for all $x, y, z \in S$.

\section{On the Ideals of Extended Special Subsets in Implicative Semigroups}

Bang and So [1] defined a subset of an implicative semigroup $S$ as follows. For any elements $x_{1}, \cdots, x_{n} \in S$ and $n \in \mathbb{N}$, it is defined a set $S\left(x_{1}, \cdots, x_{n}\right):=$ 
$\left\{z \in S \mid \Pi_{i=1}^{n} x_{i} * z=1\right\}$ which is called an extended special set. Here $\prod_{i=1}^{n} x_{i} * z:=$ $x_{1} *\left(x_{2} *\left(\cdots *\left(x_{n} * z\right)\right) \cdots\right)$. Related to the extended special set, we obtain the following theorems.

Theorem 4.1. Let I be a non empty subset of a left distributive implicative semigroup $S$. Suppose that $x_{1} \leq x_{2} \leq \cdots \leq x_{n}$. If $I$ is an ideal of $S$ then $S\left(x_{1}, \cdots, x_{n}\right) \subseteq I$ for all $x_{1}, x_{2}, \cdots, x_{n} \in I$.

Proof. Assume that $I$ is an ideal of $S$ and let $x_{1}, x_{2}, \cdots, x_{n} \in I$. If $z \in$ $S\left(x_{1}, \cdots, x_{n}\right)$ then $x_{1} *\left(x_{2} *\left(\cdots *\left(x_{n} * z\right)\right) \cdots\right)=1$. Using Proposition 2.1 and Definition 2.3, we obtain

$$
\begin{aligned}
z & =1 * z=\left(x_{1} *\left(x_{2} * \cdots *\left(x_{n} * z\right) \cdots\right)\right) * z \\
& =\left(x_{1} *\left(x_{2} * \cdots *\left(\left(x_{n-1} * x_{n}\right) *\left(x_{n-1} * z\right)\right) \cdots\right)\right) * z \\
& =\left(x_{1} *\left(x_{2} * \cdots *\left(1 *\left(x_{n-1} * z\right)\right) \cdots\right)\right) * z=\left(x_{1} *\left(x_{2} * z\right)\right) * z \in I .
\end{aligned}
$$

Hence $S\left(x_{1}, \cdots, x_{n}\right) \subseteq I$.

Proposition 4.2 ([1]). Let $S$ be a commutative left distributive implicative semigroup and $x_{1}, x_{2}, \cdots, x_{n} \in S$. If $x_{1} \leq x_{2} \leq \cdots \leq x_{n}$ then $S\left(x_{1}\right)=S\left(x_{1}, x_{2}\right)=\cdots=S\left(x_{1}, x_{2}, \cdots, x_{n}\right)$.

Theorem 4.3. Let $S$ be a commutative left distributive implicative semigroup. If $I$ is an ideal of $S$ and $x_{1} \leq x_{2} \leq \cdots \leq x_{n}$ then $I=\cup_{x_{i} \in I} S\left(x_{1}, \cdots, x_{n}\right)$.

Proof. Suppose $x, x_{1}, \cdots, x_{n-1} \in I$. Since $S$ is commutative, by Proposition $2.2, x_{1} *\left(x_{2} *\left(\cdots\left(x *\left(\cdots\left(x_{n-1} *\left(x_{n} * x\right)\right) \cdots\right)\right) \cdots\right)\right)=\cdots=x_{1} *\left(x_{2} *\right.$ $\left.\left(\cdots *\left(x_{n-1} *(x * x)\right)\right) \cdots\right)=1$. By definition, $x \in S\left(x_{1}, \cdots, x, \cdots, x_{n-1}\right)$ and

$$
S\left(x_{1}, \cdots, x, \cdots, x_{n-1}\right) \subseteq \cup_{x_{1}, \cdots, x, \cdots, x_{n-1} \in I} S\left(x_{1}, \cdots, x, \cdots, x_{n-1}\right) .
$$

Therefore $I \subseteq \cup_{x_{i} \in I} S\left(x_{1}, \cdots, x_{n}\right)$.

Conversely, let $y \in \cup_{x_{i} \in I} S\left(x_{1}, \cdots, x_{n}\right)$. Then there are $x_{1}^{\prime}, \cdots, x_{n}^{\prime} \in I$ with $x_{1}^{\prime} \leq x_{2}^{\prime} \leq \cdots \leq x_{n}^{\prime}$ such that $y \in S\left(x_{1}^{\prime}, \cdots, x_{n}^{\prime}\right)=S\left(x_{1}^{\prime}, x_{2}^{\prime}\right)=S\left(x_{1}^{\prime}\right)$ by Proposition 4.2. This means, $1 *\left(x_{1}^{\prime} * y\right)=x_{1}^{\prime} * y=1 \in I$. Therefore by Theorem 2.4, we obtain $y=1 * y \in I$. Hence $I \supseteq \cup_{x_{i} \in I} S\left(x_{1}, \cdots, x_{n}\right)$. 


\section{Necessary and Sufficient Condition for a Fuzzy Set to be a Fuzzy Ideal}

In this section we give an equivalent condition for a fuzzy implicative ordered filter to be a fuzzy ideal.

Theorem 5.1. Let $S$ be a commutative implicative semigroup and $\mu$ be a fuzzy implicative ordered filter. Then $\mu$ is a fuzzy ideal of $S$ if and only if

$$
\mu(x * y) \geq \mu(y) \quad \text { for every } x, y \in S . \quad(\star)
$$

Proof. Let $\mu$ be a fuzzy ideal of $S$ and $x, y, z \in S$. Then

$$
\mu(x * z) \geq \min \{\mu(x *(y * z)), \mu(y)\} .
$$

Choose $y=z$, using Proposition 2.1 we obtain

$$
\begin{aligned}
\mu(x * z) & \geq \min \{\mu(x *(z * z)), \mu(z)\}=\min \{\mu(x *(1)), \mu(z)\} \\
& =\min \{\mu(1), \mu(z)\}=\mu(z) .
\end{aligned}
$$

Conversely, let $\mu$ be a fuzzy implicative ordered filter satisfying ( $\star$ ), then for all $x, y, z \in S$,

$$
\begin{aligned}
\mu(x * z) & \geq \min \{\mu(x *(y * z)), \mu(x * y)\} \\
& \geq \min \{\mu(x *(y * z)), \mu(y)\} .
\end{aligned}
$$

Therefore $\mu$ is a fuzzy ideal.

An interesting result is that a fuzzy ideal is equivalent to a fuzzy ordered filter. This is stated in the following theorem.

Theorem 5.2. Let $S$ be a commutative implicative semigroup. A fuzzy set $\mu$ is a fuzzy ideal if and only if $\mu$ is a fuzzy ordered filter.

Proof. Suppose that $\mu$ is a fuzzy ideal. Then for all $x, y, z \in S$

$$
\mu(x * z) \geq \min \{\mu(x *(y * z)), \mu(y)\} .
$$

Put $x=1$. We get

$$
\begin{aligned}
\mu(z)=\mu(1 * z) & \geq \min \{\mu(1 *(y * z)), \mu(y)\} \\
& =\min \{\mu(y * z), \mu(y)\} .
\end{aligned}
$$


Hence $\mu$ is a fuzzy ordered filter. Conversely, assume that $\mu$ is a fuzzy ordered filter, and using Proposition 2.2, for every $x, y, z \in S$

$$
\begin{aligned}
\mu(x * z) & \geq \min \{\mu(y *(x * z)), \mu(y)\} \\
& =\min \{\mu(x *(y * z)), \mu(y)\} .
\end{aligned}
$$

Therefore $\mu$ is a fuzzy ideal.

Jun[5] have proved the following theorem.

Theorem $5.3([5])$. Let $\mu$ be a fuzzy set in a commutative implicative semigroup $S$. Then $\mu$ is a fuzzy ordered filter of $S$ if and only if $x \cdot y \leq z$ implies $\mu(z) \geq \min \{\mu(x), \mu(y)\}$ for all $x, y, z \in S$.

Finally, we obtain the following remark.

Remark 5.4. Let $\mu$ be a fuzzy set in a commutative implicative semigroup S. $\mu$ is a fuzzy ideal if and only if $y \leq x * z$ (or $x \leq y * z)$ implies $\mu(z) \geq$ $\min \{\mu(x), \mu(y)\}$ for all $x, y, \in S$.

\section{Conclusion}

We have proved necessary conditions for a non empty subset of an implicative semigroup to be an ideal. On the other hand, we obtained that a fuzzy ideal of an implicative semigroup is equivalent to a fuzzy ordered filter and some conditions.

\section{References}

[1] K. Bang and K. S. So, Extended Special Sets in Implicative Semigroups,Commun. Korean Math. Soc. 22, No 1 (2007), 9-14.

[2] M. W. Chan and K. P. Shum, Homomorphism of Implicative Semigroups, Semigroups Forum, 46, (1993), 7-15.

[3] Y.B. Jun and K.H. Kim, Positive Implicative Ordered Filters of Implicative Semigroups, Int. J. Math. Math. Sci.. 23, No. 12 (2000), 801806.

[4] Y.B. Jun and K. H. Kim., On Ideals of Implicative Semigroups, International Journal of Mathematics and Mathematical Sciences, 27, No.2 (2001), 77-82. 
[5] Y.B. Jun, Fuzzy Implicative Ordered Filters in Implicative Semigroup, Southeast Asian. Bull. Math., 26, (2003), 935-943.

[6] S.W. Kuresh, Y.B. Jun and W. Huang. Fuzzy Ordered Filters in Implicative Semigroups. Chinese Quart. J. Math., 13, No.2 (1998), 53-57.

[7] Nemitz, Implicative Semi-Lattices, Trans. Amer. Math. Soc., 117, (1965), 128-142. 
